\title{
Cardiac amyloidosis evaluation by bedside ultrasound in the emergency department. A case report.
}

\author{
Elyse Lavine ${ }^{1}$, Sebastian D. Siadecki ${ }^{1}$, Resa E. Lewiss ${ }^{1}$, Sarah E. Frasure ${ }^{2}$, Turandot Saul ${ }^{1}$
}

'Department of Emergency Medicine, Emergency Ultrasound Division, Mount Sinai St. Luke's / Roosevelt Hospital Center, New York, New York, USA, ${ }^{2}$ Department of Emergency Medicine, Brigham and Women's Hospital, Boston, Massachusetts, USA.

\begin{abstract}
Cardiac amyloidosis is a disease process of abnormal amyloid fibril deposition in cardiac muscle that can be diagnosed by specific electrocardiographic and echocardiographic findings. We present the case of a patient with a history of cardiac amyloidosis who presented to the emergency department (ED) with shortness of breath, and was diagnosed with a large pericardial effusion by ultrasonography. The patient's myocardium exhibited a granular, "sparkling" appearance with increased echogenicity, typical for cardiac amyloidosis. We discuss the bedside ultrasound findings characteristic of this infiltrative cardiomyopathy, and how they influenced the ED management and treatment plan for this patient.
\end{abstract}

Keywords: bedside ultrasound, amyloidosis, cardiac ultrasound

\section{Introduction}

Amyloidosis is a disease process defined by the clinically significant deposition of abnormal insoluble amyloid fibrils within tissues such as cardiac muscle. Although there are currently no non-invasive tests that can diagnose cardiac amyloidosis, characteristic electrocardiographic and echocardiographic findings are highly suggestive of the disease [1]. Amyloidosis is classified based on the protein that makes up the majority of the deposits. Senile systemic amyloidosis is a variant in which transthyretin is deposited in the heart and presents as a slowly progressive infiltrative cardiomyopathy. This particular variant is predominantly found in individuals over 60 years of age.

Received 20.10.2013 Accepted 12.12.2013

Med Ultrason

2014, Vol. 16, No 1, 63-66

Corresponding author: Turandot Saul, MD RDMS, RDCS

St. Lukes / Roosevelt Hospital Center

Department of Emergency Medicine

1000 Tenth Avenue, New York,

New York 10019

Phone: 917-209-5110

E-mail: turan@joshsaul.com
Cardiac amyloidosis leads to a restrictive cardiomyopathy that is evidenced by slow ventricular filling, and abnormal heart muscle relaxation [2]. Patients with this disease typically have significant diastolic dysfunction accompanied by signs of right-sided heart failure. The ventricular and septal walls become markedly thickened and increasingly non-compliant, a hallmark of the disease on echocardiography [3]. The autopsy report of a patient with cardiac amyloidosis reveals pronounced thickening of the myocardial walls [4].

\section{Case report}

A 67-year-old female presented with one day of acute onset shortness of breath, a syncopal episode, and declining mental status. The patient had a past medical history significant for diabetes, hypertension, atrial fibrillation, congestive heart failure, and a recent diagnosis of senile systemic amyloidosis. The patient stated that the only symptoms of the amyloidosis she had previously experienced were pain and parasthesias from peripheral neuropathy. Increasing amounts of oxygen delivered to the patient by her home oxygen system provided minimal improvement in her symptoms. 
In the resuscitation room, the patient was only able to speak in one-word sentences. Her oxygen saturation was $84 \%$ on a $100 \%$ non-rebreather mask. Other vital signs included a blood pressure of $112 / 92 \mathrm{mmHg}$, a heart rate of 122 beats $/ \mathrm{min}$, and a respiratory rate of 35 breaths $/ \mathrm{min}$. On physical examination, the patient's heart rhythm was irregularly irregular, and there was evidence of jugular venous distension. The breath sounds were diminished bilaterally, and the patient was using accessory muscles to breathe. The patient had bilateral 4+ symmetric pitting edema of her lower extremities and anasarca.

The emergency physician initially suspected acute pulmonary edema. As a result, the patient was immediately given 3 sub-lingual nitroglycerin tablets. The patient was also placed on bi-level positive airway pressure BiPAP and given $40 \mathrm{mg}$ of intravenous furosemide. An electrocardiogram (ECG) demonstrated atrial fibrillation with a rapid ventricular response rate of 144 beats $/ \mathrm{min}$, low voltage QRS complexes and a non-specific intraventricular conduction delay (fig 1). The patient was given $10 \mathrm{mg}$ of diltiazem intravenously, with a subsequent

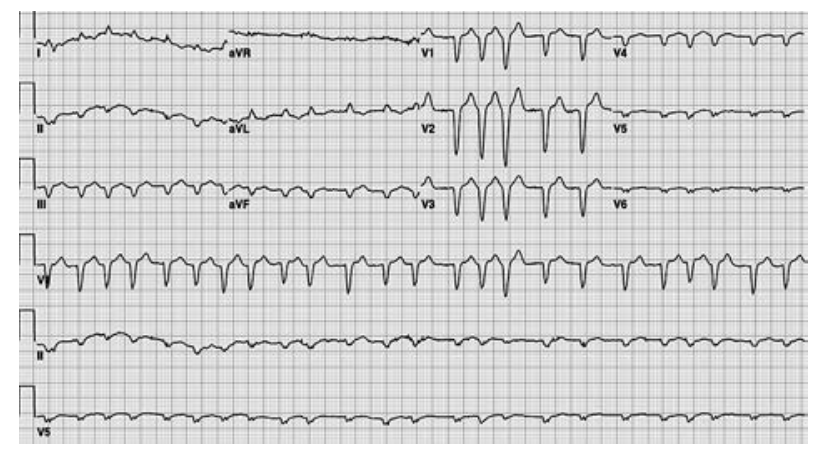

Fig 1. Electrocardiogram demonstrating atrial fibrillation with a rapid ventricular response, low voltage QRS complexes and a non-specific intra-ventricular conduction delay

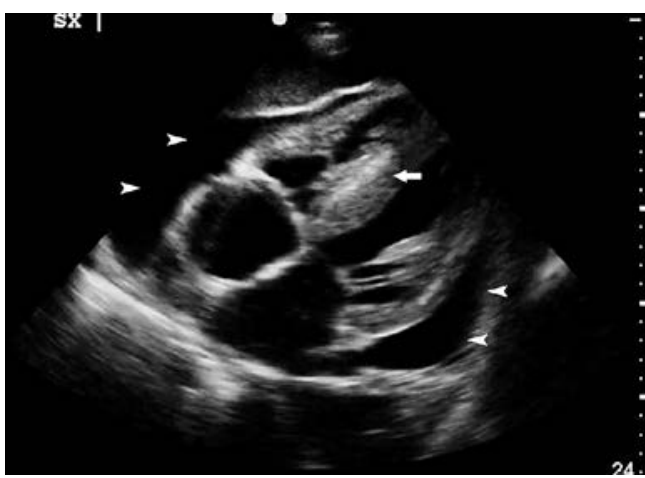

Fig 2. Subxiphoid view of the heart demonstrating thickened ventricular walls and intraventricular septum (arrow) and a large pericardial effusion (arrowheads) improvement in her heart rate to 99 beats/min. Clinician performed ultrasound imaging was obtained using a SonoSite M-Turbo ultrasound machine (Bothell, WA) with a 5-1 MHz phased array transducer placed in the sub-xiphoid (fig 2), parasternal long axis (fig 3) and parasternal short axis (fig 4) locations. The emergency physician visualized right and left ventricular hypertrophy with poor left ventricular systolic function, and a large pericardial effusion. The myocardium demonstrated a granular appearance. There was no right ventricular diastolic collapse. As the patient remained normotensive in the emergency department and expressed significant improvement in her respiratory symptoms after she was placed on BiPAP, an emergency pericardiocentesis was deferred. A portable anterior-posterior chest radiograph subsequently demonstrated nearly complete opacification of the left hemi-thorax (fig 5).

A cardiology consultation was obtained, and admission to the cardiac intensive care unit, along with aggressive diuresis was recommended. Computerized tomography of the chest was later performed to evaluate for a

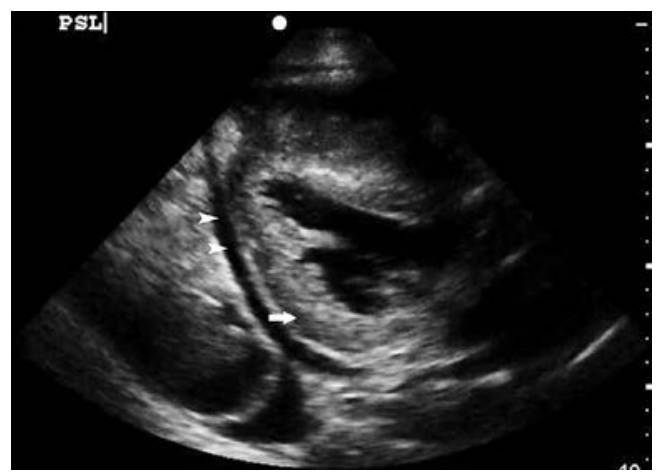

Fig 3. Para-sternal long axis view of the heart demonstrating a thickened left ventricular wall (arrow) and a pericardial effusion (arrowheads)

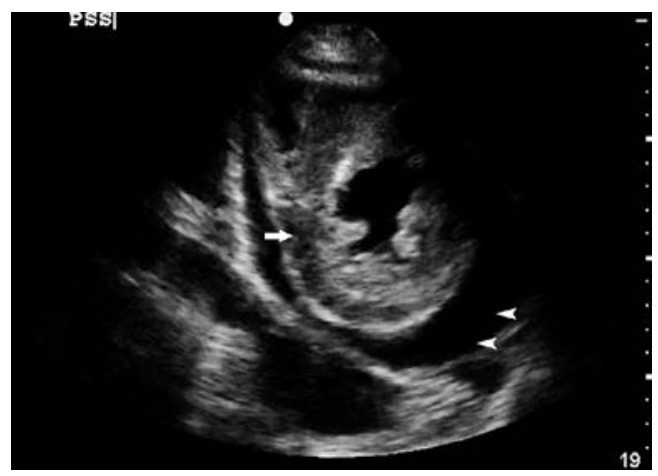

Fig 4. Para-sternal short axis view of the heart demonstrating a thickened left ventricular wall (arrow) and a pericardial effusion (arrowheads) 


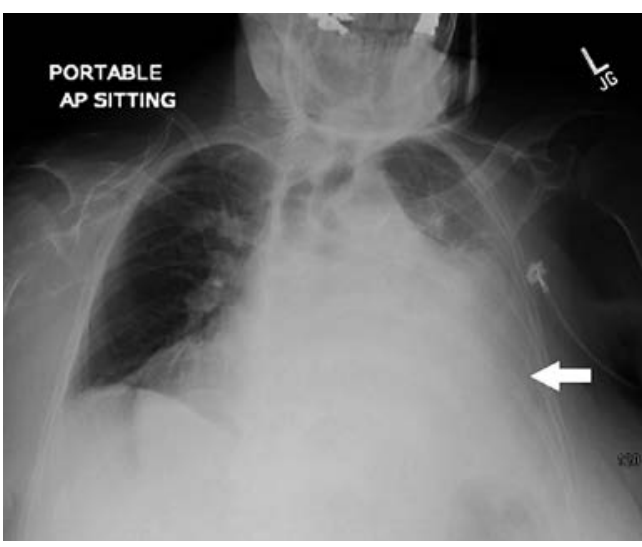

Fig 5. Anterior-posterior chest radiograph demonstrating nearly complete opacification of the left hemi-thorax (arrow)

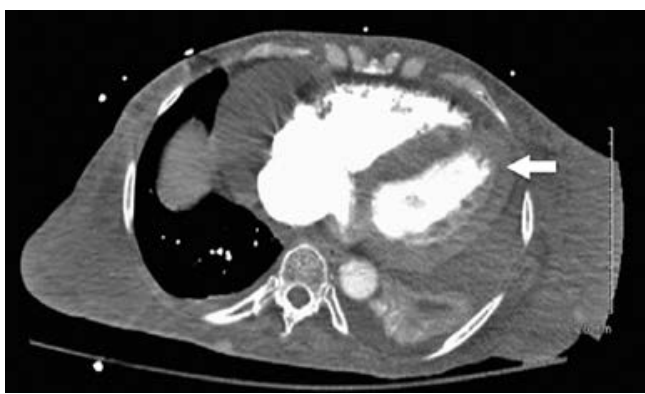

Fig 6. Computerized tomography of the chest demonstrating a pericardial effusion (arrow), cardiomegaly, and a left pleural effusion

pulmonary embolism. The findings of this study included: a large pericardial effusion, cardiomegaly, a left pleural effusion and consolidation of the middle and lower lobes of the left lung (fig 6). The patient was admitted to the cardiac care unit for diuresis and close monitoring. She was eventually discharged with home hospice care.

\section{Discussion}

We present the case of a patient with decompensated heart failure due to a restrictive cardiomyopathy from cardiac amyloidosis. The characteristic ultrasonographic finding of amyloidosis is a granular, "sparkling" myocardial appearance with increased echogenicity [1,5-8]. Patients typically have concentric bi-ventricular wall thickening with non dilated ventricles [9]. The thickened ventricular walls, in the absence of a history of hypertension, are suggestive of an infiltrative disease. The physician may also note thickening of the mitral valve leaflets if they are infiltrated with amyloid $[4,10]$. Some patients may have bilateral atrial dilation but this is not specific to amyloidosis $[4-5,10]$. Commonly the atrial septum will become thickened as well [9].

Diastolic dysfunction is the hallmark of the disease process, but as in this case, systolic dysfunction can be present in the more severe stages of amyloidosis $[1,5,10]$. In a study by Sigueira-Filho et al it was found that twentyeight patients with cardiac amyloidosis all presented with symptoms of congestive heart failure. Furthermore, 58\% of patients were noted to have a pericardial effusion [4]. Notably, in many cases of amyloidosis, the thickening of the ventricular walls prevents diastolic collapse despite elevated intra-pericardial pressures, avoiding clinical signs of tamponade and averting the need for pericardiocentesis.

The ECG in patients with cardiac amyloidosis may demonstrate a low-voltage or a pseudo-infarct pattern (no corresponding wall motion abnormalities actually seen on ultrasound) [1,11]. Atrial fibrillation and atrial flutter are also commonly noted, as in this patient's case [11]. Increased left ventricular mass in the absence of high ECG voltages is also suggestive of an infiltrative disease such as amyloidosis [1]. Rahman et al found that a combination of low voltage on ECG with a measured intraventricular septal thickness of $>1.98 \mathrm{~cm}$ on ultrasonography could make the diagnosis of amyloidosis with a sensitivity of $72 \%$ and a specificity of $91 \%$ [5].

This patient had clinician performed ultrasonographic findings of ventricular wall thickening with granular, "sparkling" myocardium, a poor ejection fraction and a large pericardial effusion, all findings in severe cardiac amyloidosis. The emergency physician was able to implement cardiac ultrasonography to initiate appropriate therapy, guide management and obtain specialist consultation in a timely manner.

In conclusion, findings consistent with cardiac amyloidosis are recognizable by the emergency physician despite the rarity of this pathology and may assist in the care of the patient.

\section{References}

1. Selvanayagam, JB, Hawkins, PN, Paul, B, Myerson, SG, Neubauer, S. Evaluation and Management of the Cardiac Amyloidosis. J Am Coll Cardiol. 2007; 50: 2101-10.2. Arora, S, Arora, A, Makkar, RP, Monga, M. Stiff heart syndrome. CMAJ. 2003; 169: 392-3.

3. Cueto-Garcia, L, Reeder, GS, Kyle, RA, et al. Echocardiographic findings in systemic amyloidosis: spectrum of cardiac involvement and relation to survival. J Am Coll Cardiol. 1985; 6: 737-43.

4. Sigueira-Filho, AG, Cunha, CL, Tajik, AJ, Seward, JB, Schattenberg, TT, Giuliani, ER. M-mode and two-dimen- 
sional echocardiographic features in cardiac amyloidosis. Circulation. 1981; 63: 188-96.5. Rahman, JE, Helou, EF, Gelzer-Bell, R, et al. Noninvasive diagnosis of biopsy-proven cardiac amyloidosis. J Am Coll Cardiol. 2004; 43: 410-5.

6. Falk RH, Plehn JF, Deering T,et al. Sensitivity and specificity of the echocardiographic features of cardiac amyloidosis. Am J Cardiol. 1987; 59: 418-22.

7. Child JS, Levisman JA, Abbasi AS, MacAlpin, RN. Echocardiographic manifestations of infiltrative cardiomyopathy. A report of seven cases due to amyloid. Chest. 1976; 70:726-31.
8. Cacoub P, Axler O, DeZuttere D, et al. Amyloid and cardiac involvement. Ann Med Interne. 2000; 151; 611-17.

9. Falk, RH. Diagnosis and management of the cardiac amyloidosis. Circulation. 2005; 112: 2047-60.

10. Simons M, Isner JM. Assessment of relative sensitivities of non-invasive tests for cardiac amyloidosis in documented cardiac amyloidosis. Am J Cardiol. 1992; 69: 425-7.

11. Murtagh B, Hammill SC, Gertz MA, Kyle, RA, Tajik, AJ, Grogan, M. Electrocardiographic findings in primary systemic amyloidosis and biopsy proven cardiac involvement. Am J Cardiol. 2005; 95; 535-7. 